Neurosurg Focus 19 (6):E11, 2005

\title{
Endoscopic third ventriculostomy
}

\author{
George I. Jallo, M.D., Karl F. Kothbauer, M.D., And I. Rick Abbott, M.D. \\ Division of Pediatric Neurosurgery, The Johns Hopkins Hospital, Baltimore, Maryland; Division of \\ Neurosurgery, Kantonsspital Luzern, Switzerland; and Department of Neurosurgery, Albert Einstein \\ College of Medicine, New York, New York
}

\begin{abstract}
The traditional treatment for all forms of hydrocephalus has been the implantation of ventricular shunt systems; however, these systems have inherent tendencies toward complications such as malfunction and infection. A significant advance in the treatment of hydrocephalus has been the evolution of endoscopy. The recent technological advances in this field have led to a renewed interest in endoscopic third ventriculostomy as the treatment of choice for obstructive hydrocephalus. Although several different endoscopes are available, the authors favor a rigid one to perform a blunt fenestration of the third ventricle floor. This description of the technique stresses the nuances for successful completion of this procedure.
\end{abstract}

KEY WORDS • endoscope • endoscopy • hydrocephalus • third ventriculostomy

\section{BRIEF HISTORY OF VENTRICULOSCOPY}

Ventriculoscopy was introduced in the early 1900s as an alternative treatment to control hydrocephalus. Walter E. Dandy was one of the first surgeons to use a primitive endoscope to perform choroid plexectomy in a patient with communicating hydrocephalus. He later introduced the subfrontal approach for an open third ventriculostomy; however, the high mortality rate and the necessity of sacrificing the optic nerve with this approach prompted Dandy to adopt a different treatment. ${ }^{1}$ The first ETV was performed by William Mixter, a urologist, in 1923. Mixter used a urethroscope to examine and perform the third ventriculostomy in a child with obstructive hydrocephalus. ${ }^{10}$ Tracy J. Putnam then borrowed this urethroscope and made the necessary modifications to optimize its use for the ventricular system. His ventriculoscope was specifically designed for cauterization of the choroid plexus in children with hydrocephalus. ${ }^{12}$ Nevertheless, the advent of valveregulated shunt systems and the simplicity of the technique resulted in minimal advances in third ventriculostomies for 30 years.

In 1947, H. F. McNickle introduced a percutaneous method of performing third ventriculostomy that decreased the complication rate and improved the success rate. ${ }^{9}$ The leukotome was introduced in the early 1970 s to enlarge the perforation in the floor of the third ventricle without injury to the surrounding vascular structures. These percutaneous techniques were then further modified after the advent of stereotactic frames. ${ }^{7}$ There has since been renewed interest

Abbreviations used in this paper: $\mathrm{BA}=$ basilar artery; $\mathrm{CSF}=$ cerebrospinal fluid; ETV = endoscopic third ventriculostomy; MR = magnetic resonance. in the use of ETV for the treatment of obstructive hydrocephalus. This has been kindled by the advent of advanced fiberoptic and lens technology. There are now small neuroendoscopes available that have deflectable tips, working ports, and good optic resolution, in addition to the rigid endoscopes with their excellent optic resolution.

In recent series of endoscopically guided third ventriculostomies performed for the treatment of obstructive hydrocephalus, success rates between 50 and $94 \%$ have been reported. ${ }^{6,711}$ The variation seen in the success rate probably reflects patient selection and differences in technique. Improvements in technique will surely come as more clinicians perform third ventriculostomies and communicate their experiences. We illustrate our technique for ETV in this article.

\section{TECHNIQUE}

\section{Patient Selection}

A patient is considered a candidate for third ventriculostomy if he or she demonstrates symptoms and signs of hydrocephalus and anatomical features amenable to a successful procedure. The patient's anatomy is delineated using preoperative MR imaging and deemed appropriate for the procedure if enlarged lateral and third ventricles are seen in conjunction with a normal-sized or small fourth ventricle. In addition, a midsagittal section should demonstrate adequate space between the BA and the clivus under the floor of the third ventricle to allow for a safe ventriculostomy. The third ventricle should be sufficiently enlarged to allow safe, limited movement of the endoscope without injury to the lateral walls of the ventricle.

Age, cause of hydrocephalus, previous shunt placement, 
or meningitis have been used by some to exclude patients from treatment with third ventriculostomy, but these are all relative contraindications. ${ }^{2: 5,5,8}$ Small or slitlike ventricles caused by previous shunt insertion clearly increase the surgical risk; however, there are now reports of third ventriculostomy being used in these cases. The effectiveness of third ventriculostomy is diminished in infants with obstructive hydrocephalus compared with patients older than 2 years of age. Again, there are now reports advocating ETV for infants younger than 6 months of age.

In our practice, relative contraindications for an ETV are infants younger than 6 months old, slitlike ventricles, very thin cortical mantle, and communicating hydrocephalus. Nevertheless, all patients presenting with hydrocephalus are considered for the procedure, especially if they have previously been treated with a shunt.

\section{Endoscopic Equipment}

Although we have several endoscope systems, we prefer to use one for all our ETVs. We recommend that the neurosurgeon be familiar and comfortable with the components of the endoscopic system (Fig. 1). Ours includes the following:

1) Endoscope. A 4.2-mm (outer) diameter Channel Neuroendoscope (Medtronic, Inc., Minneapolis, MN), a singleuse disposable endoscope with a $13-\mathrm{cm}$ rigid shaft. This scope has a $2.1-\mathrm{mm}$ working channel.

2) Control panel. A rack that consists of a video camera and optical coupler, xenon light source, video recorder, and printer.

3) Irrigation pump. We prefer to use Ringer solution as the irrigation fluid, and we prefer this solution to be slightly warmed. We rarely use continuous irrigation during surgery; if used, the flow rate is less than $15 \mathrm{ml} /$ minute.

4) Ventricular cannula. We use a No. 14 French peelaway sheath to cannulate the ventricular system. The endoscope passes easily down this sheath. It allows a pathway for egress of CSF and irrigation fluid.

5) Bugbee wire. This is a rigid wire used as a blunt probe for monopolar cautery.

6) Balloon catheter. We use a No. 3 French Fogarty balloon catheter with a small cylindrical balloon for enlargement of the fenestration in the floor of the third ventricle. We have found it prudent to check that the balloon inflates before inserting the scope into the patient.

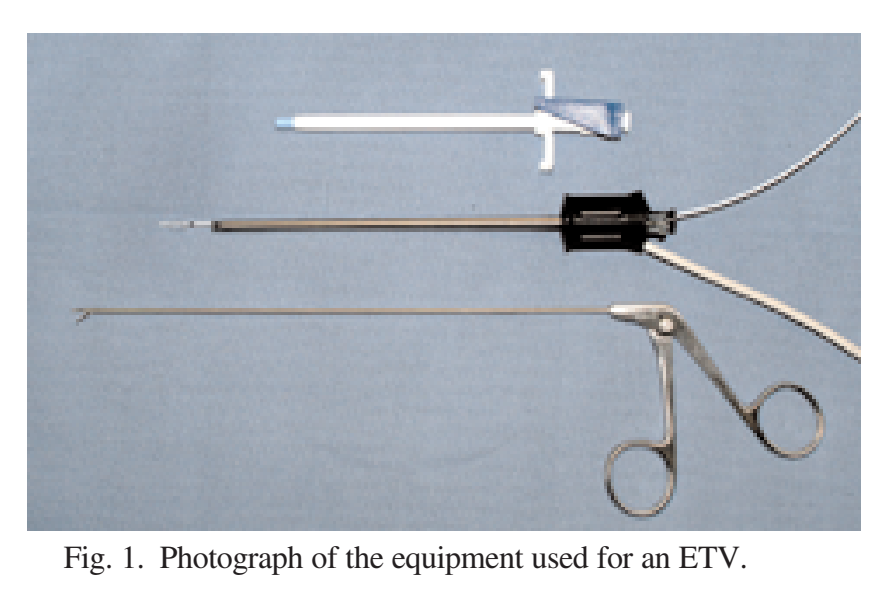

\section{Endoscopic Ventricular Aanatomy}

It is important to be familiar with the ventricular anatomy, and in particular the endoscopic anatomy, which is not three-dimensional. The foramen of Monro is often the first structure visualized. The foramina are paired structures serving to connect the lateral ventricle with the third ventricle. The head of the caudate is situated laterally and the septum pellucidum is located medially. The choroid plexus of the lateral ventricle projects forward to the foramen, through which it passes before turning posteriorly to lie under the roof of the third ventricle. The vein of septum pellucidum, located anteromedially, joins the thalamostriate vein, located posterolaterally, at the posterior rim of the foramen of Monro. These vessels join and ultimately form the internal cerebral vein, which runs in the tela choroidea of the third ventricle. These veins should become larger in caliber as they approach the foramen of Monro. The fornix is intimately related to the foramen. The fornices are paired, C-shaped, efferent-output bundles that project from the hippocampus to the mammillary bodies. They pass from the medial margin of the foramen along its anterior edge before diving into the medial wall of the third ventricle (Fig. 2).

Once the endoscope is passed into the third ventricle, there are several key anatomical landmarks. Each of the lateral walls consists of the anterior two thirds of the thalamus and hypothalamus, which is continuous with the gray matter of the floor. The lateral walls are joined to each other by a band of gray matter, the massa intermedia. The posterior border consists of the pineal body, the habenular commissure, the posterior commissure, and the cerebral aqueduct. The aqueduct is a narrow channel approximately $15 \mathrm{~mm}$ long and $1 \mathrm{~mm}$ wide that connects the third ventricle with the fourth ventricle.

A clear view of the floor of the third ventricle is provided once the endoscope is passed through the foramen of Monro (Fig. 3). The floor descends ventrally and is formed

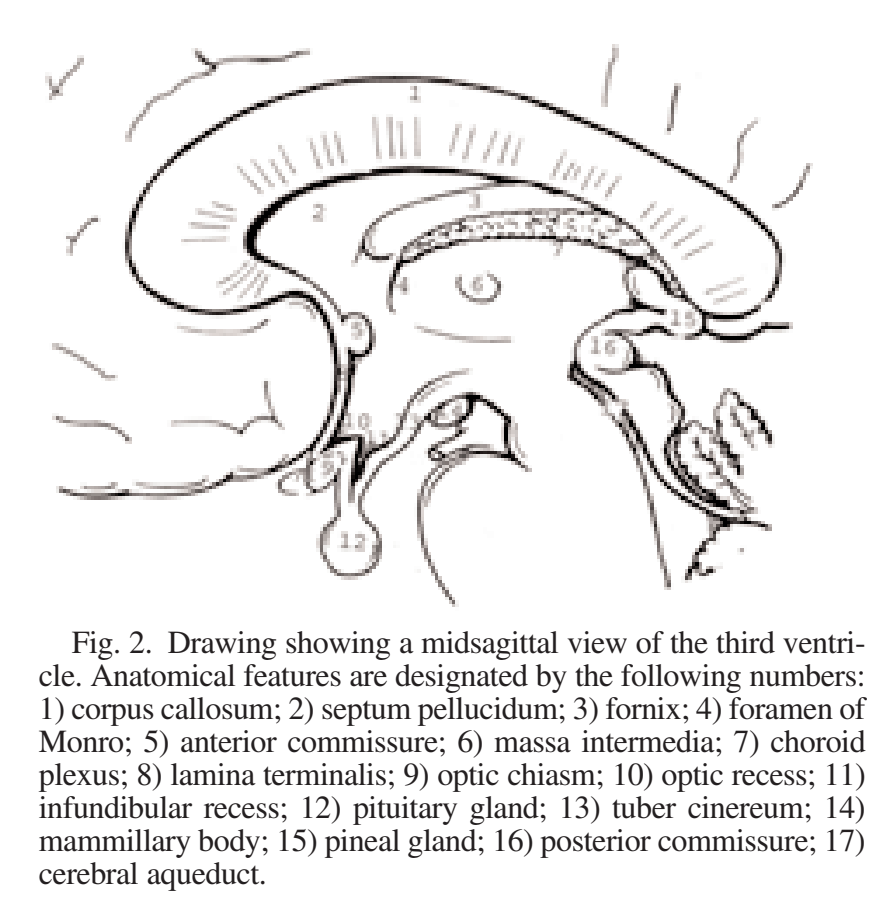


mainly by hypothalamic nuclei. There are several recesses and prominences, which serve as landmarks. In an anteroposterior direction these are the optic recess, optic chiasm, the infundibulum, infundibular recess, the tuber cinereum, and the mammillary bodies. In the majority of cases the floor (tuber cinereum) of the third ventricle is often thinned out and translucent. Posterior to the mammillary bodies is the posterior perforated substance. All these structures of the floor overlie the interpeduncular fossa.

\section{Technical Considerations}

A successful ETV depends on familiarity with the ventricular anatomy and its variations in hydrocephalus (see Video 1). After general anesthesia is induced, the patient is placed supine with the head in the neutral position on a doughnut pillow. The head is then elevated approximately $30^{\circ}$ to minimize excessive CSF loss and entry of air. A coronal burr hole is placed $3 \mathrm{~cm}$ lateral to the midline and just anterior to the coronal suture. The burr hole, which is 6 to $10 \mathrm{~mm}$ in diameter, is created on the side of the normal foramen of Monro, larger lateral ventricle, or right side. The dura mater is then opened in a cruciate fashion and the edges are coagulated. A No. 14 French peel-away catheter is then used to cannulate the lateral ventricle. This sheath is passed to lie just within the lateral ventricle. The stylet is then removed to ensure the proper placement into the ventricular system and the two leaves are peeled away and stapled to the drapes. This maneuver prevents inadvertent passage of the sheath deep into the ventricles. The advantages of this sheath include an egress pathway for irrigation fluid or CSF, and repeated passage of the endoscope without traction on or injury to the brain. The foramen of Monro is a mean distance of $6 \mathrm{~cm}$ from the dura mater via this coronal approach in an adult and less than that in children. The surgeon must be cognizant of the sheath length during cannulation of the ventricle.

Video 1. Video demonstrating the technical aspects of an ETV. (Click here to view with Windows Media Player and a broadband connection, on here to view with RealPlayer.)

Although some surgeons prefer a flexible endoscope, we prefer to use a rigid apparatus. The endoscope is passed through the sheath and the lateral ventricle is visualized. The foramen of Monro is identified, and the scope is navigated into the third ventricle. The floor of the third ventricle is, on average, $9 \mathrm{~cm}$ from the dura mater, but this is highly variable depending on age and extent of hydrocephalus. The mammillary bodies and infundibular recess are identified in the attenuated floor. It is often possible to see the BA through the diaphanous floor of the third ventricle. At this juncture, the surgeon should be certain that the intended fenestration will be anterior to the BA. It is wise to have confirmed this on the sagittal MR image obtained before surgery. A Bugbee wire, without electrocoagulation, is used bluntly to puncture the floor of the third ventricle midway between the mammillary bodies and the infundibular recess. The Bugbee wire is then removed, a No. 3 French Fogarty balloon catheter is advanced through the opening in the floor, and $0.2 \mathrm{ml}$ of fluid is instilled into the balloon, inflating it, to widen the newly created aperture. This maneuver widens the fenestration to a width of approximately $5 \mathrm{~mm}$. We do not inflate the balloon under the floor and pull back through the stoma. The scope is then carefully guided
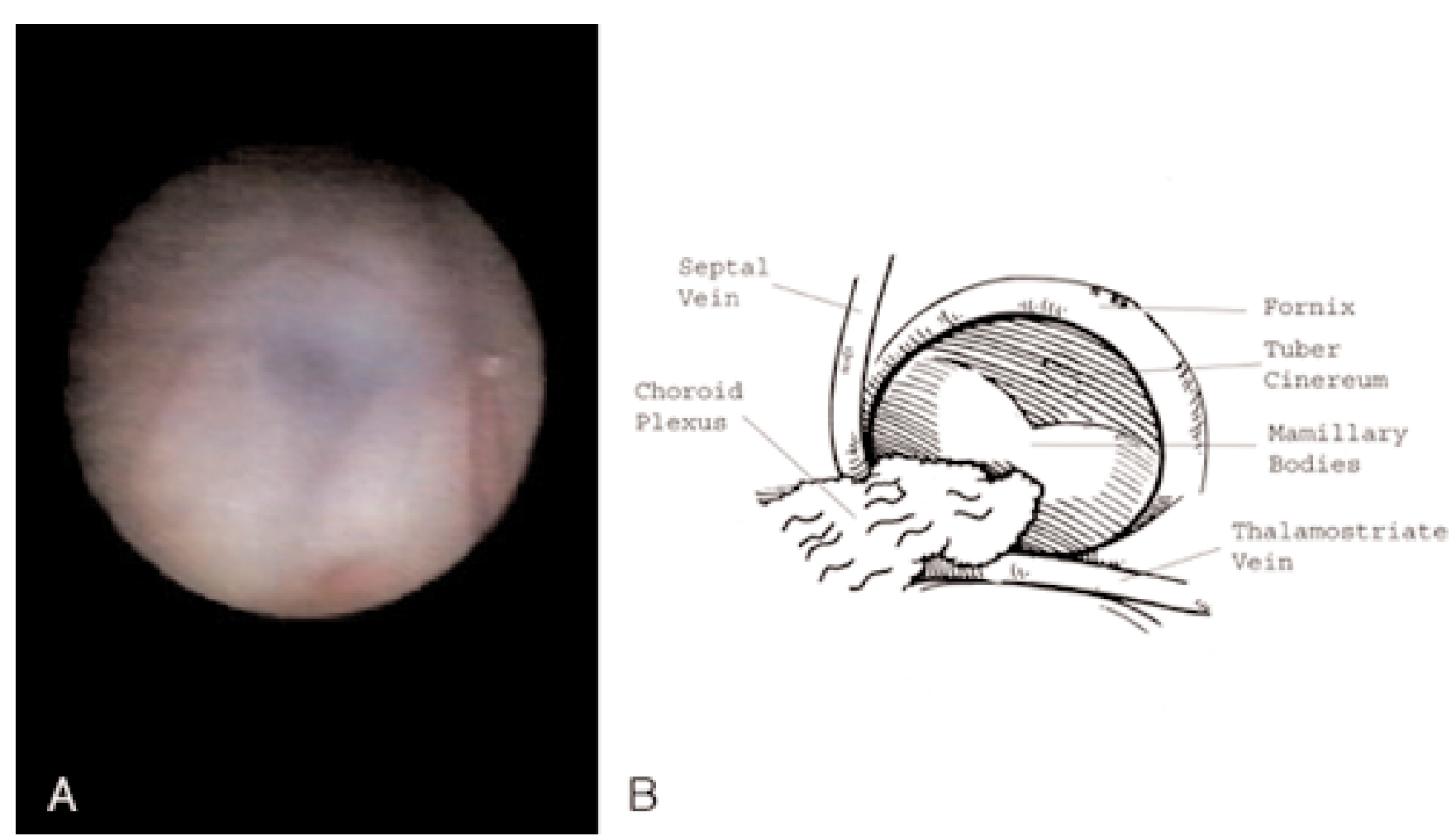

Fig. 3. A: Endoscopic view through the right foramen of Monro. B: Illustration of the endoscopically identified anatomy at the floor of the ventricle. 


\section{G. I. Jallo, K. F. Kothbauer, and I. R. Abbott}

into the prepontine cistern. Any arachnoid bands or imperforate membrane of Liliequist that seem to be impeding the free flow of CSF are bluntly disrupted with the Fogarty catheter. We do not aggressively explore this prepontine cistern for fear of injuring any perforating or branching arteries. After the stoma is created, the to-and-fro oscillations of the ventricular floor indicate good CSF communication between the ventricles and subarachnoid space.

As we became more experienced with this procedure, we gained some important insights into technical maneuvers that would enhance the safety and efficacy of the operation. One of our patients in whom treatment failed was returned to the operating room for a repeated endoscopic procedure. We observed that the previously made third ventriculostomy had remained patent. Nevertheless, there was no pulsation of fluid out of the third ventricle. After advancing the endoscope into the prepontine cistern, we observed that arachnoidal bands were preventing free CSF flow. These bands were lysed and the patient has remained shunt free. This underscores the importance of a thorough inspection and evaluation of the cisternal anatomy.

Although we use the Bugbee wire to create the fenestration, other methods include using the endoscope as a blunt trochar, and using laser, bipolar, and monopolar instruments. Doppler devices are available that can help locate the BA prior to the thermal fenestration. Nevertheless, we do not use or recommend that any energy source or the blunt scope be used to create the fenestration, for fear of injury to structures that are deep to the floor of the ventricle.

At completion of the fenestration, the endoscope and sheath are removed, Gelfoam is placed in the burr hole, and the scalp is sutured. We close the galea with a Vicryl suture and the skin with surgical staples. If any bleeding has occurred, a ventricular drain is commonly left in place for 1 to 2 days. We also leave ventricular drains in place in patients who have previously undergone shunt insertion. In these patients, we remove the shunt systems after the endoscopic procedure and observe for several days.

\section{Postoperative Care}

The patient is observed in the intensive care unit for 1 day; the total hospital stay is 2 days for the majority of patients. A postoperative computerized tomography scan is obtained prior to discharge. This study typically demonstrates an unimpressive improvement in the ventriculomegaly compared with patients in whom shunts are used. Nevertheless, the subarachnoid space and cisterns appear to be less effaced. The patient should be free of headache and ambulatory at discharge. In infants, the fontanelle should be soft and sunken while the patient is in an upright position. The ventricular drain is weaned in patients in whom shunts have previously been placed to verify shunt independence. We have left the external drain for as long as 10 days to allow the patient to tolerate the new absorption pathway. We have also performed one lumbar puncture in these patients to ensure flow through the fenestration. The drain is then removed and the patient is observed to ensure patency of the third ventriculostomy.
We obtain a follow-up MR image within 2 months of surgery; this study should demonstrate a decrease in ventricular size and/or improvement of transependymal edema. This MR image should also demonstrate a flow void in the floor of the third ventricle, or else a cine MR image is obtained for a better demonstration of CSF flow through the created fenestration.

\section{CONCLUSIONS}

Third ventriculostomy is a simple and effective procedure to control obstructive hydrocephalus. Many patients who have complicated shunt histories may become shunt independent following this procedure; however, careful patient selection and effective fenestration are key elements to its success. Although this is a safe procedure, it is associated with potentially severe complications. ${ }^{13}$

\section{References}

1. Dandy WE: An operative procedure for hydrocephalus. Johns Hopkins Hosp Bull 33:189-190, 1922

2. Drake JM: Ventriculostomy for treatment of hydrocephalus. Neurosurg Clin N Am 4:657-666, 1993

3. Guiot G: Ventriculocisternostomy for stenosis of the aqueduct of Sylvius. Puncture of the floor of the third ventricle under television control. Acta Neurochir (Wein) 28:275-289, 1973

4. Hoffman HJ, Harwood-Nash D, Gilday DL: Percutaneous third ventriculostomy in the management of noncommunicating hydrocephalus. Neurosurgery 7:313-321, 1980

5. Jaksche H, Loew F: Burr hole third ventriculocisternostomy. An unpopular but effective procedure for treatment of certain forms of occlusive hydrocephalus. Acta Neurochir (Wein) 79:48-51, 1986

6. Jones RF, Stening WA, Brydon M: Endoscopic third ventriculostomy. Neurosurgery 26:86-92, 1990

7. Kelly PJ: Stereotactic third ventriculostomy in patients with nontumoral adolescent/adult onset aqueductal stenosis and symptomatic hydrocephalus. J Neurosurg 75:865-873, 1991

8. Lapras C, Bret P, Patet JD: Hydrocephalus and aqueductal stenosis. Direct surgical treatment by interventriculostomy. J Neurosurg Sci 30:47-53, 1986

9. McNickle HF: The surgical treatment of hydrocephalus. A simple method of performing third ventriculostomy. Br J Surg 34:302-307, 1947

10. Mixter WJ: Ventriculoscopy and puncture of the floor of the third ventricle. Boston Med Surg J 188:277-278, 1923

11. Oka K, Yamamoto M, Ikeda K, et al: Flexible endoneurosurgical therapy for aqueductal stenosis. Neurosurgery 33:236-243, 1993

12. Putnam TJ: Treatment of hydrocephalus by endoscopic coagulation of the choroid plexus. N Engl J Med 210:1373-1376, 1934

13. Schroeder HWS, Niendorf WR, Gaab MR: Complications of endoscopic third ventriculostomy. J Neurosurg 96:1032-1040, 2002

Manuscript received October 20, 2005.

Accepted in final form November 21, 2005.

Address reprint requests to: George I. Jallo, M.D., Division of Pediatric Neurosurgery, The Johns Hopkins Hospital, Harvey 811, 600 North Wolfe Street, Baltimore, Maryland 21287. email: gjallo1@jhmi.edu. 